\title{
ANÁLISE DO CONSUMO DE ESTIMULANTES CEREBRAIS POR ESTUDANTES DA REGIÃO DAS MISSÓES, RIO GRANDE DO SUL, BRASIL
}

\author{
ANALYSIS OF BRAIN STIMULANT CONSUMPTION BY STUDENTS IN \\ THE REGION OF MISSÓES, RIO GRANDE DO SUL, BRAZIL
}

\author{
Kamylla Flores Silva ${ }^{1} \&$ Vera Regina Medeiros Andrade $^{1}$ \\ ${ }^{1}$ Universidade Integrada Do Alto Uruguai e Das Missões - URI Santo Ângelo, Santo Ângelo, \\ Rio Grande do Sul, Brasil.
}

\section{RESUMO}

O objetivo do estudo foi analisar o consumo de estimulantes cerebrais por estudantes. Foi realizado uma pesquisa descritiva observacional, transversal, quantitativa, com 232 universitários maiores de 18 anos, residentes na região das Missóes. O recrutamento da amostra foi por conveniência, os participantes convidados por e-mail e dados coletados por meio da plataforma "Google Forms", com questóes objetivas. Entre os participantes, a maioria era do sexo feminino $(69,4 \%)$, com faixa etária de 18 a 21 anos de idade (60,3\%), e tinha 6 a 8 horas de sono (78\%). Dos participantes, $19,9 \%$ já utilizaram algum estimulante cerebral durante a vida, e $9,5 \%$ mais de 10 vezes. O metilfenidato foi utilizado por $9,1 \%(21 / 231)$, modafinil por $1,7 \%(4 / 232)$ e piracetam por $0,4 \%(1 / 232)$ dos participantes, e $35,3 \%(82 / 232)$ costumavam ingerir bebidas energéticas. O cansaço foi relatado por 23,4\% (45/192) como motivo para uso de estimulantes, seguido de sobrecarga em práticas diárias por 16,7\% (32/192). Concluímos que a maioria era do sexo feminino, faixa etária de 18 a 21 anos de idade, com 6 a 8 horas de sono por dia. Metilfenidato, modafinil e piracetam foram os medicamentos consumidos entre os participantes com o intuito de diminuir o cansaço e sobrecarga em práticas diárias.

Descritores: Estudantes; Estimulantes do Sistema Nervoso Central, Bebidas Energéticas.

\section{ABSTRACT}

The aim of the study was to analyze the consumption of brain stimulants by students. A descriptive, observational, cross-sectional, quantitative research was carried out with 232 students over 18 years of age, residing in the region of Missoes. The sample was recruited by convenience, with participants invited by email and data collected through the "Google Forms" platform, with objective questions. Among the participants, most were female (69.4\%), aged between 18 and 21 years old (60.3\%), and had 6 to 8 hours of sleep (78\%). Of the participants, 19.9\% had already used some brain stimulant during their lifetime, and 9.5\% more than 10 times. Methylphenidate was used by $9.1 \%$ (21/231), modafinil by $1.7 \%$ (4/232) and piracetam by $0.4 \%$ (1/232) of the participants, and $35.3 \%$ (82/232) ) used to drink energy drinks. Tiredness was reported by $23.4 \%$ (45/192) as a 
reason for using stimulants, followed by overload in daily practices by 16.7\% (32/192). We concluded that the majority were female, aged between 18 and 21 years old, with 6 to 8 hours of sleep per day. Methylphenidate, modafinil and piracetam were the medications used by the participants in order to reduce fatigue and overload in daily practices.

Descriptors: Students; Central Nervous System Stimulants, Energy Drinks.

\section{INTRODUÇÁO}

Os estimulantes cerebrais são capazes de alterar ou aumentar o desempenho cerebral por meio da estimulação de neurotransmissores, que podem ocorrer naturalmente ou serem ativados sinteticamente, atuando de forma excitatória ou inibitórias ${ }^{1}$. São usados, frequentemente, para obter uma resposta cognitiva positiva, que é definida pela ingestão de substâncias para aumentar funções mentais como memória, atenção, concentração, vigília, raciocínio e pensamentos. As principais substâncias utilizadas como estimulantes do sistema nervosos central são: cloridrato de metilfenidato (Ritalina ${ }^{\oplus}$, Concerta $^{\oplus}$ ), modafinil (Stavigile ${ }^{\oplus}$, Provigi ${ }^{\oplus}$ ), piracetam $\left(\right.$ Nootropil $\left.^{\oplus}\right)$ e bebidas energéticas ${ }^{2}$.

O cloridrato de metilfenidato, que tem como principais nomes comerciais Ritalina ${ }^{\oplus}$ e Concerta ${ }^{\circledast}$ possuem mecanismo de ação estimulando o sistema nervoso central (SNC) ao bloquear a receptação da dopamina e noradrenalina ${ }^{3,4}$. Esse medicamento é indicado para o transtorno do déficit de atenção com hiperatividade (TDAH). Porém, passou a ser utilizado por atletas e estudantes universitários, de forma não recomendada, para melhorar o desempenho em avaliações e aumentar a capacidade de aprendizagem, memória e atenção de acadêmicos ${ }^{5}$. Tanto em doses recomendadas, como no consumo excessivo pode apresentar efeitos colaterais, tais como: hipertensão arterial sistêmica, redução do apetite, dificuldade em adormecer, nervosismo, náusea e boca seca ${ }^{6}$.

O modafinil, nomes comerciais de Stavigile ${ }^{\oplus}$ Provigil ${ }^{\oplus}$, é um medicamento que consiste em aumentar a liberação de dopamina e noradrenalina, serotonina e glutamato, diminuir a liberação de GABA em diversas regióes cerebrais, provocando uma relação direta ao estado de alerta, que tem como resultado a ampliação do poder de concentração e a diminuição da necessidade do sono. Estudos demostram que o modafinil melhora atividades cognitivas, a atenção, memória e facilita a aprendizagem ${ }^{1,7,8}$.

O piracetam, que tem como nome comercial de Nootropil ${ }^{\oplus}$, é indicado para tratamento sintomático da síndrome psico-orgânica, e ajuda a melhorar a perda de memória e alteraçóes da falta de direção. Seu mecanismo de ação, não está totalmente compreendido, parece que ele influência nas funçóes cognitivas, neuronais e vasculares envolvidas nos processos de aprendizagem, memória, transmissão e plasticidade sináptica, proliferação e migração celular. 
É um fármaco que apresenta poucos relatos de efeitos adversos, porém ocasionalmente pode ocorrer ansiedade, agitação, insônia, irritabilidade e tremor ${ }^{6,9}$.

As bebidas energéticas foram produzidas com o intuito de aumentar a resistência física, proporcionar reaçóes mais rápidas e maior concentração, aumentar o estado de alerta mental, evitar o sono, proporcionar sensação de bem-estar e estimular o metabolismo. O uso das mesmas estáo cada vez mais comuns entre os jovens, sendo que eles têm acesso livre a elas, podendo encontrar em qualquer barzinho, festas, academias, porém podem ser prejudiciais à saúde quando consumidas em excesso ${ }^{10}$.

O uso excessivo e inapropriado de estimulante cerebrais vem preocupando, uma vez que pode colocar a saúde em risco. Esses medicamentos são substâncias de controle especial, no Brasil, conforme a Portaria 344/1998 da ANVISA e suas atualizaçôes, sendo registrados e controlados no Sistema Nacional de Gerenciamento de Produtos Controlados (SNGPC) ${ }^{11,12}$. De acordo com ANVISA (2013) está ocorrendo um aumento crescente do uso do medicamento em todas as regióes do país. Conforme pesquisa realizada por Santana et al. (2020), com estudantes de ensino pré-vestibular e superior, a maioria fazia uso de algum tipo de psicoestimulante. No estudo realizado por Cordeiro e Pinto (2017) e Martello et al. (2020), os pesquisadores verificaram consumo de metilfenidato e piracetam. Diante do exposto o presente trabalho tem como objetivo analisar o consumo de estimulantes cerebrais por estudantes universitários.

\section{METODOLOGIA}

Realizou-se um estudo descritivo observacional, transversal, quantitativo com estudantes de 22 cursos de graduação de uma universidade na regiáo das Missóes, Rio Grande do Sul, Brasil, com idade igual ou maior de 18 anos. O recrutamento da amostra foi por conveniência, sendo os participantes convidados por e-mail a participarem da pesquisa.

O projeto de pesquisa foi aprovado pelo Comitê de Ética em Pesquisa da - Universidade Regional Integrada do Alto Uruguai e das Missões - URI - Campus de Santo Ângelo, sob o parecer substanciado número 4.553.585. Antes do participante responderem as perguntas do questionário, foi apresentado o termo de consentimento livre e esclarecido (TCLE) que explicava os objetivos, método e a importância do estudo, e se o participante aceitasse participar ele acessava o link do Google Forms para responder as questóes.

Coletou-se os dados por meio de um questionário, utilizando a plataforma do Google, "Google Forms", com questóes objetivas fechadas, no período de abril a maio de 2021, com um total de 232 pessoas participantes. Para caracterizar os estudantes participantes da pesquisa, esses foram questionados quanto a idade, o sexo e horas de sono. Sobre o uso de estimulantes cerebrais, os participantes foram questionados se já utilizaram algum estimulante cerebral, quantas vezes na vida fizeram uso de estimulantes e se fazem uso continuo. Também foram questionados sobre 
os tipos de estimulantes cerebrais utilizados, e os motivos pela busca desses estimulantes. Com esses dados, realizou-se uma análise descritiva.

\section{RESULTADOS}

\section{Perfil dos participantes}

Entre os 232 participantes, a maioria era do sexo feminino $(69,4 \%)$, a maioria estava na faixa etária de 18 a 21 anos de idade, e responderam ter de 6 a 8 horas de sono (Tabela 1).

Tabela 1 - Distribuição dos participantes da pesquisa conforme o perfil. Santo Ângelo, RS, Brasil. $2021(n=232)$.

\begin{tabular}{lcc}
\hline & $\mathrm{n}$ & $\%$ \\
\hline Gênero & & \\
Feminino & 161 & 69,4 \\
Masculino & 71 & 30,6 \\
Faixa etária & & \\
$18-21$ & 140 & 60,3 \\
$22-25$ & 58 & 25 \\
$26-29$ & 13 & 5,6 \\
$\geq 30$ & 21 & 9,1 \\
Horas de sono & & \\
$3-5$ & 28 & 12,1 \\
$6-8$ & 181 & 78 \\
$9-11$ & 21 & 9,1 \\
$\geq 12$ & 2 & 0,9 \\
\hline
\end{tabular}

\section{Uso de medicamentos}

Entre os 231 participantes, 30,7\% utiliza medicamentos de uso continuo. Do 231 participantes que responderam sobre os estimulantes, 19,9\% já utilizaram algum estimulante cerebral durante a vida, e 11,7\% responderam que talvez já utilizaram, 9,5\% já utilizaram mais de 10 vezes durante a vida (Tabela 2).

Tabela 2 - Distribuição dos participantes da pesquisa quanto ao uso de medicamentos. Santo Ângelo, RS, Brasil. 2021 ( $\mathrm{n}=231)$.

$\mathrm{n}$

Faz uso de medicamento continuo

Sim

Não
71

30,7

160

69,3 
Já utilizou algum estimulante cerebral

Sim

46

158

27

Talvez

Quantas vezes em sua vida utilizou estimulantes

$$
0
$$

1-3

4-6

7-9

$\geq 10$

\section{Tipos de medicamentos}

Tabela 3 - Distribuição dos tipos de medicamentos psicoestimulante utilizados entre os estudantes participantes da pesquisa. Santo Ângelo, RS, Brasil. 2021 ( $n=231 / 232)$.

\begin{tabular}{lcc}
\hline & $\mathrm{N}$ & $\%$ \\
\hline Já utilizou metilfenidato & 21 & \\
Sim & 210 & 9,1 \\
Não & 231 & 90,9 \\
Total & & 100 \\
Já utilizou modafinil & 4 & \\
Sim & 228 & 1,7 \\
Não & 232 & 98,3 \\
Total & & 100 \\
Já utilizou piracetam & 1 & \\
Sim & 231 & 0,4 \\
Não & 232 & 99,6 \\
Total & & 100 \\
\hline
\end{tabular}

Entre os participantes, $11,2 \%$ relataram já ter feito uso de algum medicamento. Questionados sobre metilfenidato, modafinil e piracetam, o primeiro foi utilizado por $9,1 \%$ (21/231), enquanto o segundo por $1,7 \%$ (4/232) e o terceiro por $0,4 \%$ dos participantes (Tabela $3)$.

\section{Bebidas energéticas}

Sobre as bebidas energéticas, 35,3\% (82/232) dos participantes costumavam ingerir bebidas energéticas, entre eles, 40,9\% ingeriam pelo menos 1 vez ao mês e 4,7\% todos os dias (Tabela 4). 
Tabela 4 - Distribuição dos participantes da pesquisa quanto ao uso de bebidas energéticas e a frequência. Santo Ângelo, RS, Brasil. 2021 ( $\mathrm{n}=232)$.

\begin{tabular}{lcc}
\hline & $\mathrm{n}$ & $\%$ \\
\hline Costuma tomar bebidas energéticas & & \\
Sim & 82 & 35,3 \\
Não & 150 & 64,7 \\
Com que frequência ingere bebidas energéticas & & \\
Nunca & 89 & 38,4 \\
Todos os dias & 11 & 4,7 \\
Toda semana & 37 & 15,9 \\
Uma vez ao mês & 95 & 40,9 \\
\hline
\end{tabular}

\section{Motivos}

Quando questionados sobre os motivos para uso de estimulantes, o cansaço foi relatado por 23,4\% (45/192), seguido de sobrecarga em práticas diárias por 16,7\% (32/192) (Tabela 5).

Tabela 5 - Distribuição dos participantes da pesquisa conforme os motivos pela busca de estimulantes cerebrais. Santo Ângelo, RS, Brasil. 2021 ( $\mathrm{n}=192)$.

\begin{tabular}{lcc}
\hline & $\mathrm{N}$ & $\%$ \\
\hline Motivo pela busca dos estimulantes cerebrais & & \\
Sono excessivo & 21 & 10,9 \\
Falta de concentraçáo & 26 & 13,5 \\
Sobrecarga em práticas diárias & 32 & 16,7 \\
Cansaço & 45 & 23,4 \\
Outro & 68 & 35,4 \\
\hline
\end{tabular}

\section{DISCUSSÃO}

Os 232 participantes da presente pesquisa eram em sua maioria do sexo feminino (69,4\%), na faixa etária de 18 a 21 anos de idade (60,3\%) e responderam ter de 6 a 8 horas de sono (78\%). Esses dados estão semelhantes aos encontrados por Cordeiro e Pinto $(2017)^{1}$, que realizaram pesquisa com acadêmicos da área da saúde na cidade de Ponta Grossa-PR; com Masini et al. (2019) ${ }^{15}$, que pesquisaram entre estudantes de medicina de uma faculdade do oeste da Bahia; com Morgan et al. (2017) ${ }^{2}$, que analisaram estudantes de medicina de uma universidade do extremo sul do Brasil; com Menezes et al. $(2017)^{16}$, que estudaram acadêmicos de uma instituição de ensino superior do estado de Rondônia e com o estudo de Mazzoglio et al. $(2011)^{17}$ com estudantes de anatomia normal humana da faculdade de medicina da Argentina, em que os pesquisadores encontraram percentuais de participação feminina variando de $60,2 \%$ a $72 \%$. 
Difere da pesquisa realizado por Santana et al. (2020), que avaliaram estudantes, em instituiçóes de ensino pré-vestibular e superior, da cidade de Montes Claros, MG, que entre os 348 participantes, o sexo masculino representou 52,9\%. Essa diferença pode ser explicada pelo fato de que Santana et al. (2020) ${ }^{14}$ selecionaram estudantes de pré-vestibular e acadêmicos de direito, medicina e engenharia civil, sendo pessoas em duas situaçóes diferentes. Da mesma forma, se difere de Pasquini $(2015)^{18}$, que realizou um estudo entre 710 estudantes, residentes no estado de São Paulo, com homens representando 65,1\%. Essa diferença pode ser entendida devido ao fato de os participantes dessa pesquisa serem alunos de cursinho, e de escola particular e pública que iriam prestar vestibular.

Ao verificar o uso de medicamentos entre os 232 participantes do nosso estudo, 69,3\% não utilizavam medicamentos de uso continuo, e 19,9\% responderam já ter utilizado algum estimulante cerebral durante a vida, e entre esses, 9,5\% já utilizaram mais de 10 vezes ao decorrer de suas vidas. Quanto ao uso de estimulantes cerebrais, nossos dados se assemelham ao estudo de Martello et al. $(2020)^{6}$, que analisaram o uso de estimulantes cerebrais, metilfenidato, piracetam e Ginkgo Biloba em uma instituição de ensino superior no município de Rolim de Moura, RO, e observaram uma menor frequência de respostas "Sim" (28\%) quando comparado as respostas "Não" (72\%) para o uso desses. Já, com relação ao uso de medicamentos de uso contínuo, nossos dados são semelhantes aos da pesquisa realizada por Santana et al. $(2020)^{14}$, em que a maioria dos estudantes de ensino pré-vestibular $(59,6 \%)$ e superior $(76,7 \%)$ não utiliza medicação de uso diário. Porém, diferem quanto ao uso de estimulantes cerebrais, em que a maioria dos estudantes de pré-vestibular e metade dos estudantes de ensino superior faziam uso de algum tipo de psicoestimulante, com índices de 75,0\% e 50,0\%, respectivamente.

Também difere quanto ao uso de estimulantes cerebrais aos dados das pesquisas de Silveira et al. $(2015)^{19}$ e Araújo $(2017)^{20}$, que encontraram índices de 57\% e 73\%, respectivamente. Essa diferença pode ser explicada pela seleção da amostra dos diferentes estudos. Enquanto, no presente estudo, todos os acadêmicos dos diferentes cursos da universidade foram convidados a participarem, na pesquisa de Silveira et al. ${ }^{19}$ foram apenas acadêmicos do curso de medicina e na pesquisa de Araújo et al. ${ }^{20}$ foram estudantes do curso de farmácia e um dos critérios de inclusão nesses estudos era fazer uso de substâncias estimulantes.

A semelhança sobre os medicamentos de uso contínuo pode ser explicada devido a pesquisa ser voltada a estudantes, que foram identificados como a maioria jovens entre 18 e 25 anos, pois geralmente pessoas mais jovens não costumam utilizar medicamento de uso contínuo, sendo mais comum o uso desses entre idosos, hipertensos e diabéticos. Por outro lado, a diferença para o uso de estimulantes cerebrais pode ser explicada pelo fato de pré-vestibulandos sofrerem mais pressão e cobrança para ingressar em uma universidade, o que pode levar os mesmo a procurarem recursos náo recomendados para conseguirem permanecer por mais tempo estudando e focados. 
Após questionar os participantes sobre quais tipos de estimulantes cerebrais são utilizados, 11,2\% dos participantes do presente estudo, relataram já ter feito uso de algum dos medicamentos, sendo o metilfenidato 9,1\% (21/231), modafinil 1,7\% (4/232) e piracetam 0,4\% (1/232). Esses dados são semelhantes a Martello et al. $(2020)^{6}$, que obtiveram consumo de $19,05 \%$ de metilfenidato e 4,76\% de piracetam e Cordeiro e Pinto (2017) ${ }^{1}$, que avaliaram os hábitos de consumo de estimulantes cerebrais em acadêmicos da área da saúde das faculdades particulares na cidade de Ponta Grossa, PR, e observaram um índice de 2\% de piracetam e 5,3\% de metilfenidato. Essa porcentagem de consumo de estimulantes cerebrais pode ser considerada alta, pois os mesmos são de difícil acesso e necessitam de receita de controle especial para aquisição.

Sobre as bebidas energéticas, 35,3\% (82/232) dos participantes do presente estudo costumavam ingerir bebidas energéticas, entre eles, 40,9\% ingeriam pelo menos 1 vez ao mês e $4,7 \%$ todos os dias. Esses dados se aproximam aos encontrados por Morgan et al. $(2017)^{2}$, que realizaram uma pesquisa com estudantes de medicina de uma universidade do extremo sul do Brasil, e observaram que, dos 200 questionários aplicados, 38\% dos participantes responderam que utilizavam bebidas energéticas. $\mathrm{O}$ maior consumo de bebidas energéticas comparado ao consumo de medicamentos estimulantes cerebrais pode ser explicado pelo fato de que bebidas energéticas são de venda livre, podendo ser encontradas no comércio, em bares, festas, academias ${ }^{10}$.

Para os motivos pelo uso de estimulantes, o cansaço foi relatado por 23,4\% (45/192) dos participantes do nosso estudo, seguido de sobrecarga em práticas diárias por 16,7\% (32/192). Nossos dados são compatíveis com os dados da pesquisa de Morgan et al. (2017) ${ }^{2}$, em que os principais motivos alegados foram compensar a privação de sono $(47,4 \%)$ e melhorar raciocínio, atenção e/ou memória (31,6\%).

Embora as perguntas formuladas e as alternativas para as respostas das pesquisas fossem diferentes, os efeitos buscados pelos estudantes da presente pesquisa se assemelham aos estudos de Santana et al. (2020) $)^{14}$, Masini et al. (2019) ${ }^{15}$, Cordeiro e Pinto (2017) ${ }^{1}$ e Pasquini (2015) ${ }^{18}$. Na pesquisa realizada por Santana et al., a maioria dos estudantes tanto pré-vestibulandos e estudantes da graduaçáo responderam que os efeitos produzidos pelos medicamentos estimulantes era a melhora na concentração e redução do sono. No estudo de Masini et al. ${ }^{15}$ a motivação mais frequente que levou ao consumo de estimulantes cerebrais relatada pela maioria dos estudantes (71\%) foi aumentar a capacidade cognitiva. Da mesma forma, no estudo de Cordeiro e Pinto (2017) ${ }^{1}$, a motivação para o consumo destas substâncias por estudantes foi aumentar a capacidade cognitiva. Na pesquisa realizada por Pasquini ${ }^{18}$, foi relatado por todos os participantes (100\%) que o motivo do uso de estimulantes foi o aumento de rendimento escolar. O fato de os participantes estarem submetidos a uma maior pressão para disputarem uma vaga no ensino superior, ou estudantes do ensino superior se sentirem pressionados por inúmeros trabalhos acadêmicos, provas, atividades extras, pode acabar estimulando os mesmo a buscarem por estes estimulantes para aprimorarem o desempenho, concentração e falta de atenção. Outro aspecto 
importante a ser ressaltado é o fato da procura por algo físico para a solução dos problemas pela maioria das pessoas, provavelmente devido a uma questão mercadológica e comportamental observada nos tempos atuais.

\section{CONCLUSÃO}

Baseado nos dados do presente estudo, concluímos que a maioria era do sexo feminino, estava na faixa etária de 18 a 21 anos de idade, e tinha de 6 a 8 horas de sono por dia. Com relação a medicamentos, a maioria não utiliza medicamentos de uso continuo. Os participantes relataram utilizar estimulantes cerebrais como metilfenidato, modafinil e piracetam (19,9\%) e bebidas energéticas $(35,3 \%)$ com o intuito de diminuir o cansaço e sobrecarga em práticas diárias.

Uma das limitaçôes do estudo foi não ter questionado sobre diagnóstico de Transtorno do Déficit de Atenção com Hiperatividade (TDHA) e se o uso de estimulantes era com prescrição, sendo que sugerimos novas pesquisas abordando também estas questôes.

\section{REFERÊNCIAS}

1. Cordeiro N, Pinto RMC. Consumo de estimulantes cerebrais em acadêmicos da área da saúde na cidade de Ponta Grossa-PR. Rev Visão Acadêmica. 2017 Abr-Jun; 18(2): $23-45$.

2. Morgan HL, Petry AF, Licks PAK, Ballester AO, Teixeira KN, Dumith SC. Consumo de Estimulantes Cerebrais por Estudantes de Medicina de uma Universidade do Extremo Sul do Brasil: Prevalência, Motivação e Efeitos Percebidos. Rev Bras de Ed Méd. 2017 Jan-Mar; 41(1): 102-9.

3. Cesar ELR, Wagner GA, Castaldelli-Maia JM, Silveira CM, Andrade AG, Oliveira LG. Uso prescrito de cloridrato de metilfenidato e correlatos entre estudantes universitários brasileiros. Rev Psiq Clín. 2012; 39(6): 183-8.

4. Andrade LS, Gomes AP, Nunes AB, Rodrigues NS, Lemos O, Rigueiras PO, et al. Ritalina uma droga que ameaça a inteligência. Rev Med Saúde Brasilia. 2018 Maio 20; 7(1): 99-112.

5. Cândido RCF, Perini E, Pádua CM, Junqueira DR. Prevalência e fatores associados ao uso de metilfenidato para neuroaprimoramento farmacológico entre estudantes universitários. Einstein (São Paulo). 2019 out 24; 18: 1-7.

6. Martello AT, Freitas TMFS, Figueiredo TS, Rolim ELG, Benati MAFNO. O uso de metilfenidato, piracetam e ginkgo biloba - estimulantes cerebrais - por estudantes 
de uma instituição de ensino superior de Rolim de Moura (RO). Revista saberes da faculdade de São Paulo - FSP. 2020 Jun; 13(1): 1-36.

7. Garcia VA. Investigação do potencial uso do Modafinil como agente melhorador da memória [Tese]. Porto Alegre: Pontifícia Universidade Católica do Rio Grande do Sul; 2013.

8. Fernandes HA. Efeitos do modafinil sobre o aprendizado e a memória de camundongos avaliados na esquiva discriminativa em labirinto em cruz elevado [Tese]. São Paulo: Universidade Federal de São Paulo; 2011.

9. Winnicka K, Tomasiak M, Bielawska A. Piracetam--an old drug with novel properties? Acta Pol Pharm. 2005 Sep-Oct; 62(5): 405-9.

10. Ballistreri MC, webster CMC. O uso de bebidas energéticas entre estudantes de educação física. Revista Latino-am Enfermagem. 2008 Mai-Jun; 16: 1-8.

11. BRASIL. Ministério da Saúde. Secretaria de Vigilância em Saúde. PORTARIA No 344, DE 12 DE MAIO DE 1998(*). Aprova o Regulamento Técnico sobre substâncias e medicamentos sujeitos a controle especial. Disponível em: https://bvsms. saude.gov.br/bvs/saudelegis/svs/1998/prt0344_12_05_1998_rep.html. Acesso em: 24 jun 2021 .

12. BRASIL. Ministério da Saúde. Secretaria de Vigilância em Saúde.

Resolução RDC no 22, de 29 de abril de 2014. Disponível em: http://portal.crfsp.org. br/325-legislacao/5508-resolucao-rdc-22-de-29-de-abril-de-2014-anvisa.html\#. Acesso em: 24 jun 2021.

13. BRASIL. Agência Nacional de Vigilância Sanitária (ANVISA). Prescrição e consumo de metilfenidato no Brasil: identificando riscos para o monitoramento e controle sanitário. Boletim de Farmacoepidemiologia, v. 2, n.2, p. 1-14, 2012. Disponível em: https://www.anvisa.gov.br/sngpc/boletins/2012/boletim_ sngpc_2_2012_corrigido_2.pdf. Acesso em: 24 Jun 2021.

14. Santana LC, Ramos AN, Azevedo BL, Neves ILM, Lima MM, Oliveira MVM. Consumo de Estimulantes Cerebrais por Estudantes em Instituiçóes de Ensino de Montes Claros/MG. Revista brasileira de educação médica. 2020 Mar 30; 44(1): 1-8.

15. Masini LD, Dantas LWP, Nunes LO, Santos MA, Rego TM, Stefanelo ST. Análise do consumo de estimulantes cerebrais por estudantes de medicina de uma faculdade do oeste da Bahia. 170 Congresso de Iniciação Científica, 2019, Barreiras Ba, UNIFASB, 2019, p. 1-5.

16. Menezes ASS, Nomerg KO, Lenzi RV. O uso de psicoestimulantes por acadêmicos de uma instituição de ensino superior do estado de Rondônia. Repositório Institucional FACIMED, 2017; 1-13. 
17. Mazzoglio y Nabar MJ, Algieri RD, Dogliotti CB, Gazzotti AM, JimenezVillarruel HN, Rey LM. Utilización de sustancias psicoactivas en alumnos de anatomía y su implicación en el aprendizaje. Educ Med. 2011; 14 (2): 129-32.

18. Pasquini NC. Fármacos para turbinar o cérebro, uso por quem pretende entrar na universidade. Eletronic Journal of Pharmacy. 2015; 12(3): 36-42.

19. Silveira VI, Oliveira RJF, Caixeta MR, Andrade BBP, Siqueira RGL, Santos GB. Uso de psicoestimulantes por acadêmicos de medicina de uma universidade do sul de minas gerais. Rev Univ Vale do Rio Verde. 2015; 13(2): 186-92.

20. Araujo JS. Consumo de estimulantes cerebrais por estudantes de farmácia da universidade federal da paraíba: prevalência, motivaçáo e efeitos percebidos [monografia]. João Pessoa: Universidade Federal da Paraíba; 2019.

21.

Autor Correspondente: Kamylla Flores Silva E-mail: kamyllafsilva@aluno.santoangelo.uri.br Recebido em: 2021-07-20 Aprovado em: 2021-09-14 